\title{
Analytical Approximation Algorithm for the I nverse of the Power of the Incomplete Gamma Function Based on Extreme Value Theory
}

\author{
Shanshan $\mathrm{Wu}^{1}$, Guobing $\mathrm{Hu}^{2 *}$, Li Yang ${ }^{2}$ and Bin $\mathrm{Gu}^{1}$ \\ ${ }^{1}$ School of Electronic Information Engineering, Nanjing Vocational College of Information Technology, Nanjing, \\ Jiangsu, 210023, China \\ [e-mail: wuss@njcit.cn] \\ ${ }^{2}$ School of Electronic and Information Engineering, Jinling Institute of Technology, Nanjing, Jiangsu, 211169, \\ China \\ [e-mail: s0304152@jit.edu.cn] \\ *Corresponding author: Guobing $\mathrm{Hu}$
}

Received January 5, 2021; revised March 7, 2021; accepted December 1, 2021;

published December 31, 2021

\begin{abstract}
This study proposes an analytical approximation algorithm based on extreme value theory (EVT) for the inverse of the power of the incomplete Gamma function. First, the Gumbel function is used to approximate the power of the incomplete Gamma function, and the corresponding inverse problem is transformed into the inversion of an exponential function. Then, using the tail equivalence theorem, the normalized coefficient of the general Weibull distribution function is employed to replace the normalized coefficient of the random variable following a Gamma distribution, and the approximate closed form solution is obtained. The effects of equation parameters on the algorithm performance are evaluated through simulation analysis under various conditions, and the performance of this algorithm is compared to those of the Newton iterative algorithm and other existing approximate analytical algorithms. The proposed algorithm exhibits good approximation performance under appropriate parameter settings. Finally, the performance of this method is evaluated by calculating the thresholds of space-time block coding and space-frequency block coding pattern recognition in multiple-input and multiple-output orthogonal frequency division multiplexing. The analytical approximation method can be applied to other related situations involving the maximum statistics of independent and identically distributed random variables following Gamma distributions.
\end{abstract}

Keywords: incomplete Gamma function, extreme value theory, normalized coefficient, Gumbel function.

This study was supported by a research grant from Nanjing Vocational College of Information Technology [Project No. YK20200101], the Natural Science Foundation of the Jiangsu Higher Education Institutions of China [Project No. 20KJA510008], and the project of National Vocational Education Teaching Innovation Team [Project No. YB2020080102]. 


\section{Introduction}

In recent years, the inverse of the incomplete Gamma function has been increasingly utilized in wireless communication signal processing. The solution of certain problems such as the calculation of the secure outage probability of multiple-input and multiple-output (MIMO) multi-antenna selection systems [1], error probability analysis of M-ary orthogonal signals in fading channels [2], throughput analysis of maximum sum capacity channel sensing strategies in time-varying wireless channels [3], and MIMO signal pattern recognition [4-7] involves the inverse of the power of the incomplete Gamma function without exception.

However, an exact analytical solution method is yet to be devised for the problem of the inverse of the power of the incomplete Gamma function; thus, a common means of addressing such problems is to transform them into incomplete Gamma function inverse problems. The existing algorithms can be divided into two categories: algorithms based on numerical evaluation [8-9] and algorithms related to the analytical expression [10-11]. Although the algorithms based on numerical evaluation have higher accuracy, they inevitably have common shortcomings. First, a good initial approximation of the root is needed for efficiency and convergence. Second, iteration involves computational complexity, which is not conducive to hardware implementation. Obviously, the analytic expression algorithm is more interpretable, besides, it is more suitable for practical engineering applications, such as the implementation of the algorithm in digital signal processor and field-programmable gate arrays (DSP/FPGAs). However, the existing inversion methods based on analytic expressions have some limitations on parameters. For example, in [11], the lower incomplete Gamma function was represented by the generalized hypergeometric function. This approach has enabled the inverse of the Gamma function to be obtained in closed form with low complexity. However, the continuous hypergeometric function can be expanded asymptotically only when the root is small.

To overcome the aforementioned limitations, this article proposes an analytical approximation algorithm based on extreme value theory (EVT) for the inverse of the power of the incomplete Gamma function. The basic ideas are as follows: The Gumbel function was used to approximate the power of the incomplete Gamma function, and the normalized coefficient of the generalized Weibull distribution function was employed to replace the normalized coefficient of the random variable following a Gamma distribution, hence the approximate closed form solution was obtained. Through simulation under various conditions, the approximate analytical solution obtained using this algorithm was compared with the exact numerical solution based on the Newton iterative method, confirming the effectiveness of the algorithm and providing scope for other applications. In addition, the algorithm can also be applied to the inverse problem of the incomplete Gamma function after proper transformation. At the end of this study, two cases were discussed regarding the application of the algorithm for signal processing in communication. The algorithm was applied in a threshold setting of space-time block coding (STBC) and space-frequency block coding (SFBC) code pattern recognition algorithms in MIMO-orthogonal frequency division multiplexing (OFDM) systems. 


\section{Extreme Value Theory (EVT)}

\subsection{Gamma Distribution and Its Equivalence Properties}

\section{Theorem 1 (Fisher-Tippett Extremum Type Theorem)[12]}

Suppose $X_{1}, X_{2}, \ldots, X_{n}$ is an independent and identically distributed random sequence, whose distribution function is $F(x)$ and whose maximum is defined as $X_{(n)}=\max \left(X_{1}, X_{2}, \ldots, X_{n}\right)$. If constants $\left\{a_{n}>0\right\}$ and $\left\{b_{n}\right\}$ exist, let

$$
\lim _{n \rightarrow \infty} \operatorname{Pr}\left(\frac{X_{(n)}-b_{n}}{a_{n}} \leq x\right)=G(x), x \in R,
$$

where $G(x)$ is a nondegenerate distribution function. Then, its form must belong to one of the following three types of distributions:

Type I distribution (Gumbel distribution)

$$
G_{1}(x)=\exp \left\{-e^{-x}\right\}, x \in R ;
$$

Type II distribution (Frechet distribution)

$$
G_{2}(x, \beta)=\left\{\begin{array}{l}
0, x \leq 0 \\
\exp \left\{-x^{-\beta}\right\}, x>0, \beta>0
\end{array} ;\right.
$$

Type III distribution (Weibull distribution)

$$
G_{3}(x, \beta)=\left\{\begin{array}{l}
\exp \left\{-(-x)^{\beta}\right\}, x \leq 0, \beta>0 \\
1, x>0
\end{array} .\right.
$$

These three functions are called extreme value distributions, and the parameter $\beta$ is related to the tail of the probability density function $F(x)$. The Fisher-Tippett extreme type theorem shows that, for independent and identically distributed random variables, regardless of the type of probability density function, after proper normalization, the normalized extreme value random variable $\frac{X_{(n)}-b_{n}}{a_{n}}$ converges to one of the aforementioned three extreme value distribution types. This characteristic indicates that knowing the parent distribution of random variable enables the limit distribution type of its extreme value to be inferred. The three extreme value limit distributions can be unified as follows:

$$
G(x ; \mu, \delta, \xi)=\exp \left\{-\left(1+\xi \frac{x-\mu}{\delta}\right)^{-1 / \xi}\right\}, 1+\xi(x-\mu) / \delta>0, x \in R,
$$

where $\mu, \delta, \xi$ represents position parameter, scale parameter, and shape parameter, respectively.

Equation (5) is known as a generalized extreme value distribution. In practice, this distribution is often fitted by the block maxima (BM) method. For different parent distributions of the three types, the limit distribution of the extreme is decided by maximum domain attraction (MDA). Regarding the maximum value, the so-called attraction field states that

$$
\lim _{n \rightarrow \infty} \operatorname{Pr}\left(\frac{X_{(n)}-b_{n}}{a_{n}} \leq x\right)=\lim _{n \rightarrow \infty} F^{n}\left(a_{n} x+b_{n}\right)=G(x) .
$$


Then, the random variable $X$ belongs to the maximum domain of attraction of the extreme value distribution $G(x)$, which can be expressed as $X \in M D A(G)$.

Theorem 2 (Necessary and Sufficient Condition for the Maximum Domain of Attraction) [12]

For a given parent distribution, the sufficient and necessary condition ensures that the maximum limit distribution belongs to one of the three kinds of attraction fields and can be expressed as follows:

Belong to $G_{1}(x)$ if and only if

$$
\lim _{n \rightarrow \infty} n\left\{1-F\left[X_{1-1 / n}+x\left(X_{1-1 /(n e)}-X_{1-1 / n}\right)\right]\right\}=\exp (-x),
$$

where $X_{\alpha}$ is the $100 \alpha$ quantile of the parent distribution.

\subsection{Tail Equivalence of Gumbel Distribution}

\section{Theorem 3 (Tail Equivalence Property) [13]}

If the distribution functions $F(x)$ and $G(x)$ have the same right endpoint $x^{*}$, suppose that after normalization by constants $\left\{a_{n}>0\right\}$ and $\left\{b_{n}\right\}, F(x)$ belongs to the maximum domain of attraction of the Gumbel function, which can be written as follows:

$$
\lim _{n \rightarrow \infty} F^{n}\left(a_{n} x+b_{n}\right)=G_{1}(x) .
$$

Therefore, $\lim _{n \rightarrow \infty} G^{n}\left(a_{n} x+b_{n}\right)=G_{1}(x+c)$, if and only if the tails of $F(x)$ and $G(x)$ are equivalent, which can be expressed as follows:

$$
\lim _{x \rightarrow x^{*}} \frac{1-F(x)}{1-G(x)}=e^{c}
$$

where $C$ is a constant.

According to this theorem, if $c=0$, then $\lim _{x \rightarrow \infty}[1-F(x)] /[1-G(x)]=1$ is established. Thus, the tails of $F(x)$ and $G(x)$ are considered to be equivalent, and the Gumbel approximation can be conducted with the same normalized coefficient.

\section{EVT Based Algorithm for the Inverse of the Power of the Incomplete Gamma Function}

\subsection{Problem Description}

The equation involving the power of an incomplete Gamma function can be expressed as follows:

$$
P^{n}(\alpha, x)=q \quad \alpha \geq 0, x \geq 0,0 \leq q \leq 1 \quad,
$$

where $P(\alpha, x)=\frac{\gamma(\alpha, x)}{\Gamma(\alpha)}$ represents the normalized lower incomplete Gamma function, $\alpha$ is the shape parameter, $\gamma(\alpha, x)$ is the lower incomplete Gamma function, and $\Gamma(\alpha)=\int_{0}^{+\infty} t^{\alpha-1} e^{-t} d t \quad(\alpha>0)$ is the Gamma function [14]. Then, the inverse problem of the power of the incomplete Gamma function is to find $x$, given $\alpha$ and $q$ in equation (10). 


\subsection{Analytical Approximation Algorithm for the Inverse of the Power of the Incomplete Gamma Function Based on EVT}

$F(x, \alpha, \beta)$ is a Gamma distribution function with shape parameter $\alpha$ and scale parameter $\beta$. When $\beta=1, F(x, \alpha, 1)=P(\alpha, x)$. According to the order statistics theory, if $X_{1}, X_{2}, \ldots, X_{n}$ denote independent and identically distributed Gamma random vectors with shape parameter $\alpha$ and scale parameter 1 , then the distribution function of the maximum of the random sequence $X_{(n)}=\max \left(X_{1}, X_{2}, \ldots X_{n}\right)$ obeys $F^{n}(x ; \alpha, 1)=P^{n}(\alpha, x)$.

According to EVT, if $X_{1}, X_{2}, \ldots, X_{n}$ are independent identical distributed Gamma random variables with shape parameter $\alpha$ and scale parameter 1 , then the limit distribution of the distribution function of the maximum of the random variables $X_{(n)}=\max \left(X_{1}, X_{2} \mathrm{~L} X_{n}\right)$ obeys a Gumbel distribution. i.e., if suitable normalization constants $\left\{a_{n}>0\right\},\left\{b_{n}\right\}$ exist, then [15]

$$
\lim _{n \rightarrow \infty} F^{n}(x ; \alpha, 1)=\lim _{n \rightarrow \infty} P^{n}(\alpha, x)=G_{1}\left(\frac{x-b_{n}}{a_{n}}\right),
$$

where $G_{1}(x)=\exp [-\exp (-x)]$ is the Gumbel function, and the normalized coefficient is as follows [13]:

where $f(x)=\frac{d F(x ; \alpha, 1)}{d x}$ is the probability distribution function of Gamma random

$$
b_{n}=F^{-1}(1-1 / n), a_{n}=1 / n f\left(b_{n}\right),
$$
variables.

It is clear that the approximate solution becomes more accurate as $n$ increases. Based on empirical evidence, a better approximation will be obtained when $n>10$ [15].

According to equation (11), equation (10) can be transformed as follows:

$$
\exp \left[-\exp \left(-\frac{x-b_{n}}{a_{n}}\right)\right] \approx q, 0 \leq q \leq 1, n>>1 .
$$

Therefore, the approximate solution of equation (13), which can be obtained by a simple derivation, can be written as follows:

$$
x_{q}^{\mathrm{evt}} \approx-a_{n} \ln (-\ln q)+b_{n} .
$$

which is also the approximate solution of equation (10).

From equation (12), it is necessary to compute the inverse of the incomplete Gamma function to obtain the normalization coefficient, but analytic solutions do not exist. Thus, equation (14) does not provide an analytic solution. Therefore, the analytic solution of the normalization coefficient will be considered first.

Previous research has shown that the tail of the distribution of a random variable with a Gamma distribution is equivalent to that of a general Weibull distribution [14], i.e., the function of the general Weibull class distribution is as follows:

$$
G(x)=1-K x^{\lambda} \exp \left(-C x^{\tau}\right), x \geq x_{0},
$$


where $K, C, \lambda>0, \tau \geq 1$, and $x_{0} \geq 0$ are all real constants. When $K=\left[\beta^{\alpha-1} \Gamma(\alpha)\right]^{-1}$, $\lambda=\alpha-1, \mathrm{C}=1 / \beta, \tau=1$, and $x_{0}=0$, we can obtain

$$
\lim _{x \rightarrow \infty} \frac{1-F(x ; \alpha, \beta)}{K x^{\lambda} \exp (-C x)}=1 .
$$

According to the tail equivalence property of the Gumbel distribution, $F(x)$ and $G(x)$ can be approximated by Gumbel distributions with the same normalization coefficient. Hence, the normalization coefficient of a generalized Weibull distribution function can be used to replace the normalization coefficient of a random variable following a Gamma distribution. The Lamber W function was used previously [14] to obtain the approximate analytic solution of the normalization coefficients of a generalized Weibull distribution as follows,

$$
\left\{\begin{array}{l}
b_{n}^{\prime}=\beta\left\{\ln [n / \Gamma(\alpha)]+(\alpha-1) \ln B_{n}+\left[(\alpha-1)^{2} \ln B_{n}-(\alpha-1)^{2} \ln (\alpha-1)+\alpha-1\right] / B_{n}\right\} \\
a_{n}^{\prime}=b_{n}^{\prime} \beta\left[b_{n}^{\prime}+\beta(\alpha-1)\right] /\left[\left(b_{n}^{\prime}\right)^{2}-\beta^{2}(\alpha-1)(\alpha-2)\right]
\end{array} .\right.
$$

where $B_{n}=\ln [n / \Gamma(\alpha)]+(\alpha-1) \ln (\alpha-1)$.

Letting $\beta=1$, the normalization coefficients defined in equation (17) can be used to represent equation (12) and then can be substituted into equation (14) to obtain the approximate analytic solution as:

$$
x_{q}^{\mathrm{evt}} \approx-a_{n}^{\prime} \ln (-\ln q)+b_{n}^{\prime} .
$$

The calculation times of each parameter in this algorithm is analyzed in Table 1. The calculation of the parameters involves 4 types of operations, consisting of 18 addition/subtraction operations, 19 multiplication/ division operations, 8 logarithmic calculations, and 2 special functions. As the search and iteration operations are not involved in the calculation process of (18), the complexity order of the proposed algorithm is $\mathrm{O}$ (1)

\begin{tabular}{|c|c|c|c|c|}
\hline $\begin{array}{ll}\text { number of times } \\
\text { Parameters }\end{array}$ & $\begin{array}{l}\text { Addition/ } \\
\text { Subtraction }\end{array}$ & $\begin{array}{l}\text { Multiplication/ } \\
\text { Division }\end{array}$ & $\begin{array}{l}\text { Logarithm } \\
\text { Calculation }\end{array}$ & $\begin{array}{l}\text { Special } \\
\text { Function }\end{array}$ \\
\hline$B_{n}$ & 3 & 2 & 2 & 1 \\
\hline$b_{n}^{\prime}$ & 9 & 8 & 4 & 1 \\
\hline$a_{n}^{\prime}$ & 5 & 8 & 0 & 0 \\
\hline$x_{q}^{\mathrm{evt}}$ & 1 & 1 & 2 & 0 \\
\hline total & 18 & 19 & 8 & 2 \\
\hline
\end{tabular}
approximately.

Table 1. Analysis of algorithm complexity 
In summary, the approximation algorithm in this paper can be expressed as follows:

Analytical Approximation Algorithm for the Inverse of the Power of the Incomplete Gamma Function Based on EVT

Input: The parameters in equation $P^{n}(\alpha, x)=q$, such as $\alpha$, $q$, and $n$

Output: The solution $x$ of equation $P^{n}(\alpha, x)=q$.

1. Conditional testing: Check whether the parameters in equation (10) fit the scope of applications of this algorithm, that is, $\alpha \geq 0,0<q<1$, and $n>>1$.

2. Solution of normalized coefficient: Compute the normalized coefficients $a_{n}^{\prime}$ and $b_{n}^{\prime}$ in terms of the parameters $\alpha$ and $n$ in equation (17).

3. Approximate solution computation: Substitute the normalized coefficients $a_{n}^{\prime}$ and

$b_{n}^{\prime}$ into equation (18) and obtain the approximate solution of equation (10).

\section{Simulation and Analysis}

To evaluate the algorithm, its performance was simulated and analyzed under different parameter settings, as described in this section, and it has been compared to the existing approximate analytical algorithms proposed in [10], [16] and the Newton iteration method [9].

\subsection{Algorithm Performance Analysis}

This section discusses the effects of parameters $q, \alpha$, and $n$ in equation (10) on the simulation results, for performance evaluation of the proposed algorithm.

If we denote the exact numerical solution obtained by the Newton iteration method as $x_{q}^{\mathrm{ext}}$ and denote the approximate analytical solution obtained by the proposed algorithm as $x_{q}^{\mathrm{evt}}$, the relative error can be defined by,

$$
\text { err }=\left|x_{q}^{\mathrm{evt}}-x_{q}^{\mathrm{ext}}\right| / x_{q}^{\mathrm{ext}},
$$

which acts as a performance evaluation measure.

(1) Influence of $q$ on the performance of the proposed algorithm

In the equation $P^{n}(\alpha, x)=q$, let $n=1000, \alpha=\{4,6,8,10\}$, and $q=0.1 \sim 0.9$ with the step size 0.1. Fig. 1 shows the variation of the relative error, err , of the proposed algorithm with respect to the parameter $q$.

As shown in Fig. 1, when $n$ is fixed, the relative error, err , between the approximate analytical solution and the exact numerical solution decreases with increasing $q$. err is less than 6\% under different $\alpha$ when $q=0.9$, while the maximum err is $8.4 \%$ when $q=0.1$. The results show that the greater the $q$, the better the performance of the proposed algorithm. 


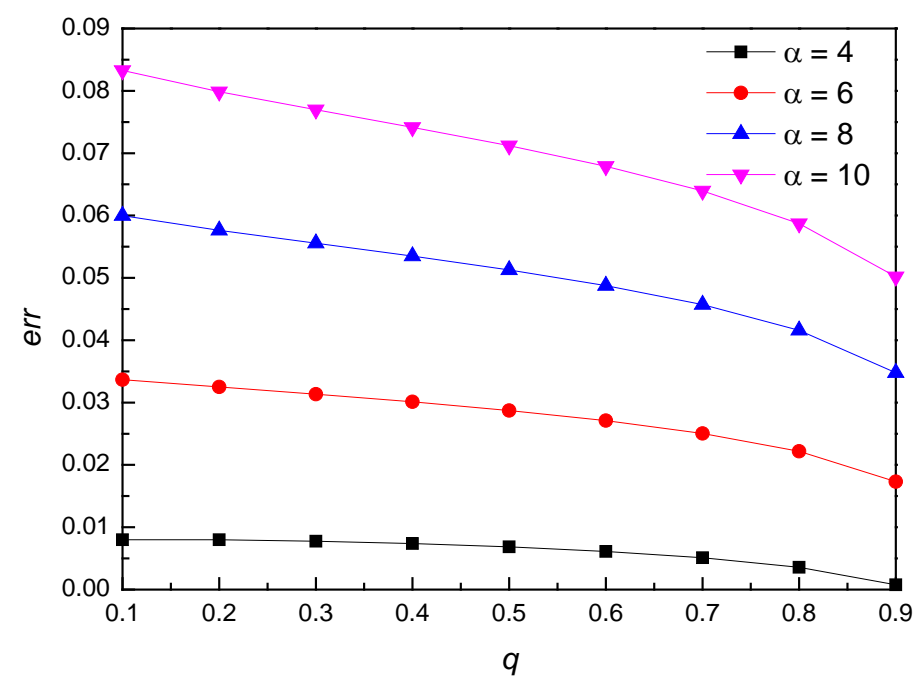

Fig. 1. Influence of $q$ on the performance of the proposed algorithm

(2) Influence of $\alpha$ on the performance of the proposed algorithm

In equation (10), when $n=1000$ and $q=\{0.9,0.99,0.999\}, \alpha$ varies from 4 to 40 with the step size 2. Fig. 2 presents the relationship between err and the parameter $\alpha$.

In the simulation, err increases with the increase of the parameter $\alpha$ when $n$ is a constant. According to [14] (Theorem 5.1 (3)), for different types of Weibull random variables, when the maximum limit distribution converges to a Gumbel distribution, the normalization constants $a_{n}$ and $b_{n}$ are:

$$
a_{n}=\frac{1}{C \tau b_{n}^{\tau-1}}+\frac{\delta}{b_{n}^{2 \tau-1}}+O\left(\frac{1}{b_{n}^{2 \tau}}\right)
$$

then,

$$
G^{n}\left(a_{n} x+b_{n}\right)=G_{1}(x)\left\{1+\left[2\left(\lambda-C^{2} \tau \delta\right) x-(\tau-1) x^{2}\right)\right] O\left(\frac{1}{b_{n}^{\tau}}\right)+O\left(\frac{1}{b_{n}^{\tau+1}}\right),
$$

where $\delta \geq 0$. As can be seen from equation (21), $\lambda$ also has some influence on the convergence rate from $G^{n}\left(a_{n} x+b_{n}\right)$ to $G_{1}(x)$. Considering the distribution function of random variables with Gamma distributions $F(x ; \alpha, \beta)$, we can obtain $\lambda=\alpha-1, C=1 / \beta, \tau=1$; therefore, the second term in equation (21) becomes

$$
\left[2\left(\alpha-1-\beta^{-2} \delta\right) x\right] O\left(\frac{1}{b_{n}}\right) \text {. }
$$

Obviously, when $\beta$ is invariant, the convergence rate decreases as $\alpha$ increases. In other words, if the thresholds of err is established, the larger $\alpha$ is, the greater is the $n$ required. 


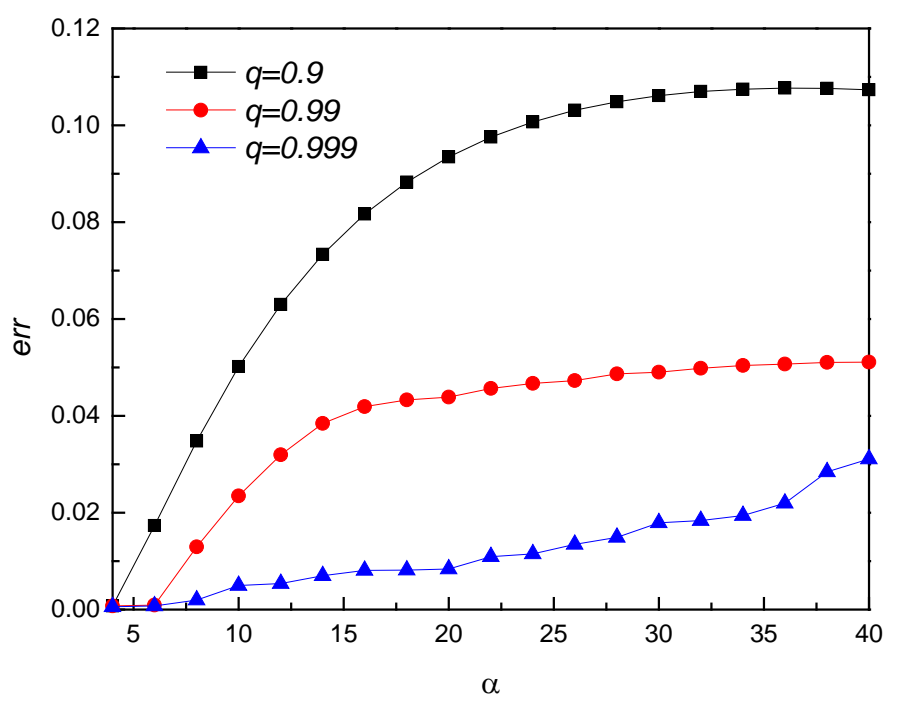

Fig. 2. Influence of $\alpha$ on the performance of the proposed algorithm

(3) Influence of $n$ on the performance of the proposed algorithm

Fig. 3 shows the performance of the proposed algorithm under different $n$, where $q=0.9$, $\alpha=\{4,6,8,10,12\}$ and $n=\{50,100,500,1000,2000\}$.

Clearly, err decreases with increasing $n$ because the normalized coefficient calculated using equation (12) is close to the approximate solution obtained using equation (17) with increasing $n$. This phenomenon is consistent with the limit properties of the coefficients under the condition of tail equivalence of different parent distribution functions in EVT, which can be represented as $\lim _{n \rightarrow \infty} a_{n} / a_{n}^{\prime}=1$ and $\lim _{n \rightarrow \infty}\left(b_{n}-b_{n}^{\prime}\right) / a_{n}^{\prime}=0[13]$.

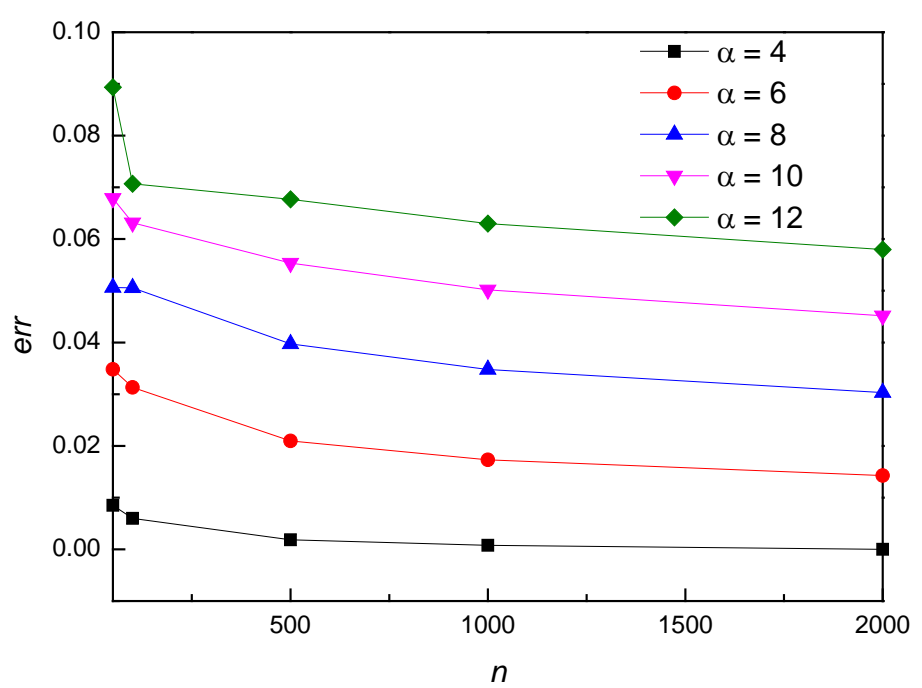

Fig. 3. Influence of $n$ on the performance of the proposed algorithm 


\subsection{Performance Comparison with Existing Algorithms}

Here, we describe the performance comparison of the proposed method with those of the methods in [10], [16], and the Newton iteration method [9]. The simulation platform was Dell (model: 131WL), and the processor was Intel (R) core (TM) i5-8265u (1.8 GHz). In the simulation, the parameters in the equation $P^{n}(\alpha, x)=q$ were set as follows: $n=1000, \alpha=3$, and $q=\{0.5,0.6,0.7,0.8,0.9\}$. Fig. 4 presents the solutions of these algorithms.

It can be observed that with moderate values of $n$ and $\alpha$, the solutions obtained using the proposed algorithm fit the exact values obtained using the Newton iterative method more closely than those determined using the algorithms in [10] and [16]. In addition, the proposed algorithm and the algorithm in [16] are greatly affected by $q$. The results of the two algorithms become closer to the exact values as $q$ increases.

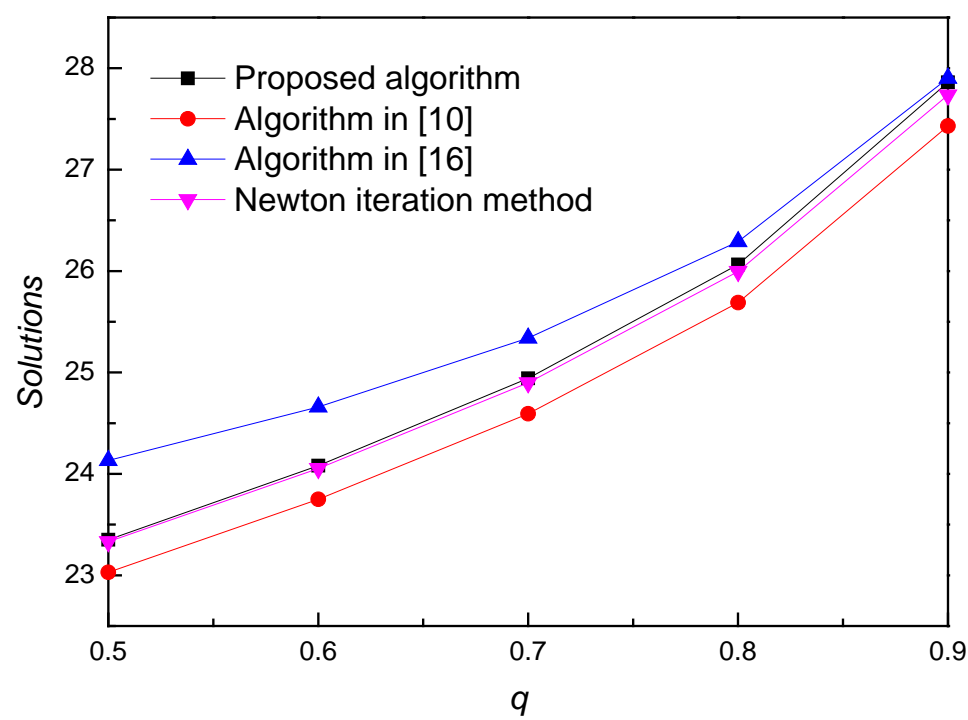

Fig. 4. Solutions of the equation versus $q$ under moderate $\alpha$ and $n$

Table 2 shows the performance and operation time of the proposed algorithm in comparison with those in [9],[10], and [16] under the same conditions. The parameters in equation (10) were set as follows: $n=1000, \alpha=3, q=0.9$, and 100 simulation experiments were conducted.

Obviously, the operation time of the proposed algorithm is slightly longer than those of the algorithms in [10] and [16] owing to the calculation of two normalization coefficients; however, the operation times of these three methods are less than that of the Newton iteration method. From the viewpoint of estimation performance, the relative error between the analytical solution obtained by this algorithm and the exact solution obtained using the Newton iteration method is only $0.24 \%$, which is less than those of the algorithms in [10] and [16]. 
Table 2. Performance and operation time comparisons between the proposed algorithm and the existing algorithms

\begin{tabular}{|c|c|c|}
\hline Algorithm & Relative error (average) & Operation time (average) \\
\hline Proposed algorithm & $0.24 \%$ & $0.18 \mu \mathrm{s}$ \\
\hline Algorithm in [10] & $1.43 \%$ & $0.08 \mu \mathrm{s}$ \\
\hline Algorithm in [16] & $0.52 \%$ & $0.15 \mu \mathrm{s}$ \\
\hline Newton iterative method [9] & - & $62.5 \mu \mathrm{s}$ \\
\hline
\end{tabular}

\section{Application Examples}

The approximate algorithm for the inverse of the power of the incomplete Gamma function studied herein is based on the extremum theory. Under certain conditions, the power of the incomplete Gamma function is approximated by a Gumbel function, simplifying the inverse operation and producing an approximate closed solution. In the calculation process, the aforementioned problem is transformed to determine the normalized coefficients of Weibull distribution functions, and an approximate analytical expression is obtained. Therefore, the approximate calculation method proposed in this paper can be applied to all relevant situations involving the maximum statistic of independent and identically distributed random variables with Gamma distributions. This section illustrates the threshold calculation in MIMO-OFDM signal pattern recognition in detail.

\subsection{Threshold Calculation for STBC Pattern Recognition in MIMO-OFDM Systems}

In the research on space-time block code recognition for MIMO-OFDM [5], the maximum delay autocorrelation function of different receiving antenna signals is considered for the recognition statistics and compared to a specific threshold to realize the recognition of spatial modulation (SM) and STBC codes. The decision statistic for code type recognition is defined as

$$
\Upsilon=\max E_{c}(\tau),
$$

where $E_{c}(\tau)=\sum_{i=0}^{N_{r}-2} \sum_{j=i+1}^{N_{r}-1} E_{i j}(\tau) . E_{i j}(\tau)$ denotes the delay cross-correlation function between the $i$-th and $j$-th receiving antennas, and $N_{r}$ represents the number of receiving antennas. As stated in [5], when SM code is selected, $E_{c}(\tau)$ approximately obeys $\chi^{2}\left(2 N_{c}\right)$ with the parameter $N_{c}=N_{r}\left(N_{r}-1\right) / 2$, which is essentially a special case of the Gamma distribution. Thus, the recognition algorithm can be concluded as: According to the constant false alarm rate (CFAR) criterion, for a given false alarm probability $P_{f a}$ and the corresponding decision threshold $\lambda$, if $\Upsilon \geq \lambda$, the code type of the received signal is determined to be STBC, otherwise it is SM. 
The decision threshold can be obtained by solving the following equation [4]:

$$
\left(1-P_{f a}\right)=\left[\frac{\gamma\left(N_{c}, \lambda / 2\right)}{\Gamma\left(N_{c}\right)}\right]^{N+v}=P^{N+v}\left(N_{c}, \lambda / 2\right),
$$

where $v$ is the cyclic prefix length of the OFDM signal, which is generally a quarter of the signal length. Obviously, it is difficult to obtain an analytical solution for equation (24); however, it can be solved by numerical methods such as Newton iteration. A numerical solution is more accurate; however, such solutions are computationally more complex, and they are not conducive to the engineering implementation of hardware platforms. Hence, in this study, we attempted to find an approximate analytical solution of equation (24) based on the proposed approximation method.

First, the applicability of the application case was evaluated. The numerical calculation and performance analysis results presented in the preceding section indicate that the approximate solution proposed in this paper has better performance when $n$ is large and $q$ approaches 1 . Considering that $n$ is generally large, when $P_{f a}$ is relatively small, $1-P_{f a}$ approaches 1 , which meets the application conditions of the proposed approximation algorithm.

Thus, the solution of equation (24) can be approximated using the following equation:

$$
P_{f a}=1-\operatorname{Pr}(\Upsilon<\lambda) \approx 1-\exp \left(-e^{-\left(\lambda-b_{n}\right) / a_{n}}\right) .
$$

By letting $\alpha=N_{c}, \beta=2, n=N+v$ and substituting these parameters into equation (17), the normalized coefficients in the equation (25) can be obtained as:

$$
\lambda \approx 2\left\{b_{N+v}-a_{N+v} \ln \left[-\ln \left(1-P_{f a}\right)\right]\right\} .
$$

Fig. 5 presents a comparison between the thresholds obtained using various methods and the exact values obtained using the Newton iterative method under different numbers of receiving antennas. In the simulation, the false alarm probability was set to 0.001 . The number of samples was 1024. The black square-symbol line in the figure is the exact threshold value obtained using the Newton iterative method, and the other lines are the approximate solutions obtained by the algorithms in [10], [16] and the proposed algorithm. It can be seen that the approximate analytical threshold obtained using the proposed algorithm is close to the exact solution. In addition, the approximate performance depends on the number of receiving antennas. As the number of receiving antennas decreases, all approximate solutions become closer to the exact solutions because the number of antennas corresponds to the shape parameters of the Gamma distribution. According to the algorithm performance analysis presented in Section 4, the approximation performance is better when the shape parameters are small. In 4G communication systems, the number of receiving antennas generally vary from 2 to 8 . 


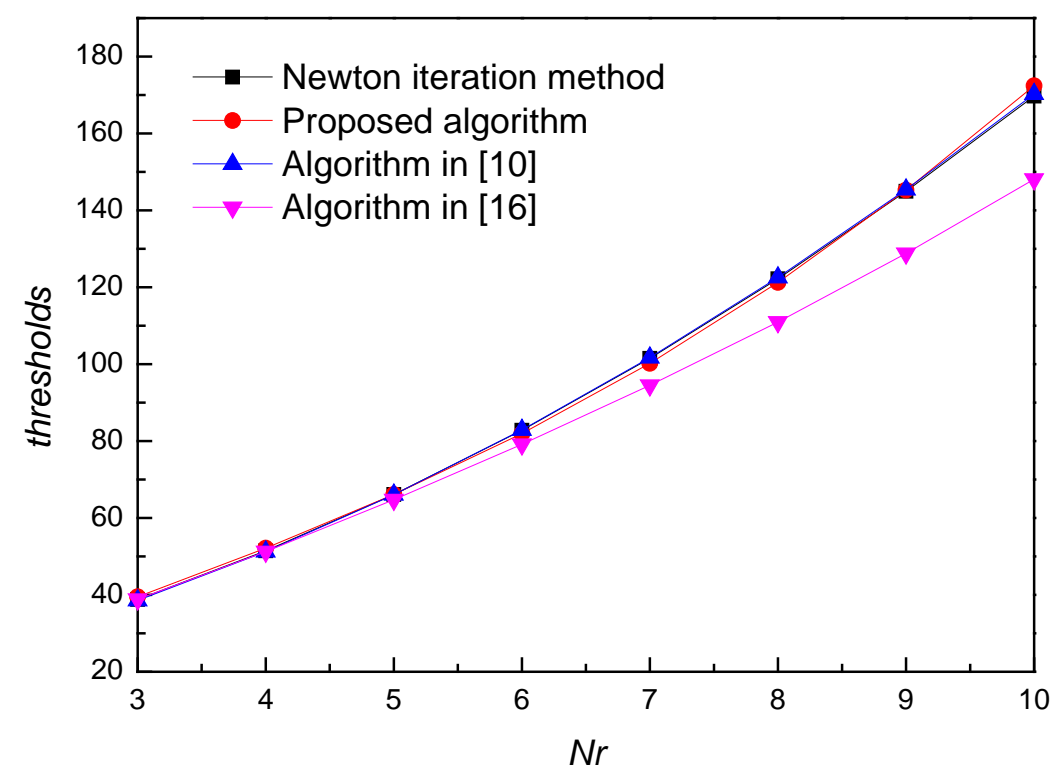

Fig. 5. Thresholds versus the number of receiving antennas for a MIMO-OFDM STBC recognition system at the false alarm probability of $0.1 \%$

\subsection{Threshold Calculation for SFBC Pattern Recognition in MIMO-OFDM Systems}

In [17], an algorithm based on the central limit theorem was proposed for the recognition of SM and Alamouti (AL)-SFBC signals in MIMO-OFDM systems. The test statistic constructed in the algorithm is,

$$
u=\sum_{i=0}^{L-1} v_{i}^{T} \hat{\psi}^{-1} v_{i}
$$

where $v_{i}=\frac{1}{\sqrt{N^{\prime} / 2}} \sum_{j=i N^{\prime} / 2+1}^{(i+1)^{N^{\prime}} / 2} r(2 j-1,2 j)$ and $\hat{\psi}=\frac{1}{N-3} \sum_{k=1}^{N-2} I_{2 D} \cdot[r(k, k+2) \operatorname{or}(k, k+2)]$ can be obtained by the cross-correlation function between the $k_{1}-$ th OFDM subcarrier at the $i$-th receiving antenna and the $k_{2}$-th OFDM subcarrier at the $j$-th receiving antenna. $L$ is the number of vectors in the group. If the number of receiving antennas is $N_{r}$, the set of receiving antennas pairs $D=N_{r}\left(N_{r}-1\right)$.

As described in [17], the recognition of SM and AL-SFBC signals can be converted into the following problem. Suppose the false alarm probability is $P_{f a}$, and the decision threshold is $\eta$. If $u \geq \eta$, the received signal is AL, otherwise it is SM.

Here, the CFAR criterion is used to determine a decision threshold $\eta$ :

$$
P_{f a}=\operatorname{Pr}(u \geq \eta) \text {. }
$$

Then, using the cumulative distribution function (CDF) expression of the chi-square distribution, we can calculate that

$$
1-P_{f a}=\operatorname{Pr}(u<\eta)=\frac{\gamma(p / 2, \eta / 2)}{\Gamma(p / 2)} .
$$


In equation (29), $\frac{\gamma(p / 2, \eta / 2)}{\Gamma(p / 2)}$ represents the normalized lower incomplete Gamma function. Let $p / 2=\alpha, \eta / 2=x$, and $Q=1-P_{f a}$; then, if the false alarm probability $P_{f a}$ is provided, the equation can be rewritten as

$$
\frac{\gamma(\alpha, x)}{\Gamma(\alpha)}=1-P_{f a}=Q .
$$

Obviously, the solution of the decision threshold $\eta$ is the inverse of the incomplete Gamma function. If $n-t h$ power is taken on both sides of equation (30), this equation can be transformed into equation (10). Inserting $q=Q^{n}$ into the equation yields

$$
\eta=2 \times\left[-\mathrm{a}_{N}^{\prime} \ln \left(-\ln \left(Q^{n}\right)+\mathrm{b}_{N}^{\prime}\right] .\right.
$$

According to this analysis, in the process of solving the decision threshold, calculating $n$-th power at both sides of equation (30) is necessary. However, there are two restrictions on the value of $n$. On one hand, in the equation $P^{n}(\alpha, x)=Q^{n}=q$, the relative error, err , between the approximate analytical solution and numerical exact solution decreases with increasing $n$, while on the other, $q$ decreases with increasing $n$. Fig. 6 compares the numerical solution of threshold $\eta$ and the approximate analytical solution obtained using the proposed algorithm with different $n$. It can be seen that when $n$ is small, the error between the approximate solution and the numerical solution decreases with increasing $n$. When $n=$ 40 , the approximate solution is close to the numerical solution. When $n$ is large, the error between the approximate solution and numerical solution increases with increasing $n$. Therefore, for practical applications, $n$ is generally set to 40 .

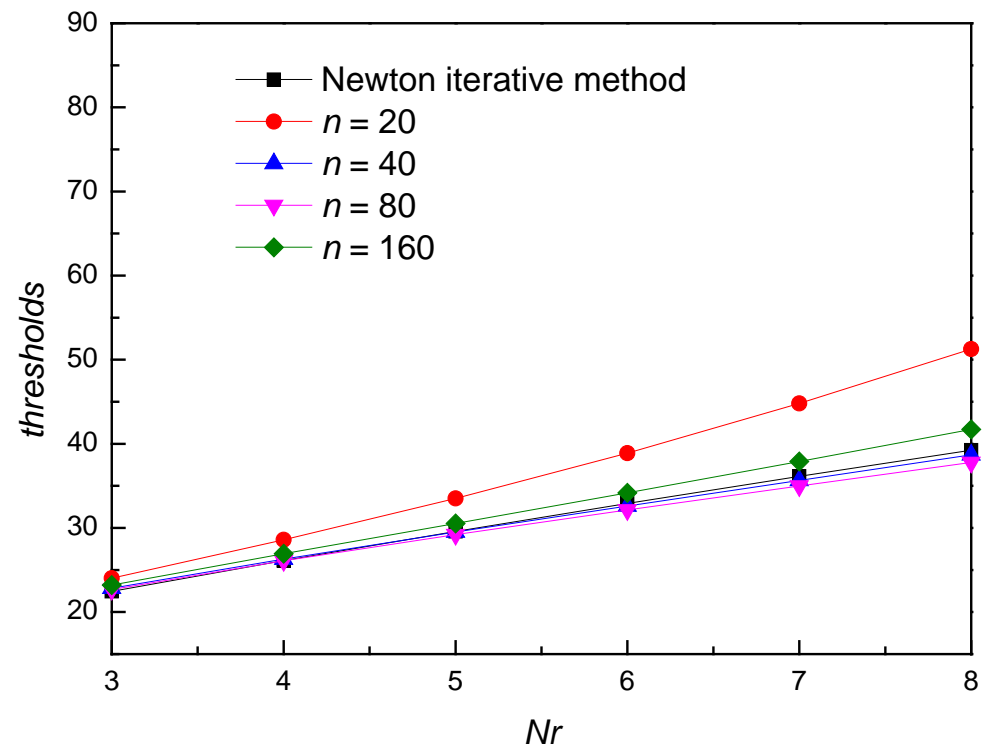

Fig. 6. Thresholds versus the number of receiving antennas for a MIMO-OFDM STBC system under different $n$ 
Focusing on the recognition threshold problem for SM and AL-SFBC signals in [17], Fig. 7 presents a performance comparison between the numerically obtained threshold and the approximate analytical solutions obtained using the proposed algorithm and those in [10] and [16]. According to the aforementioned analysis, $n=40$ and $P_{f a}=0.001$ should be used to improve the estimation performance of this algorithm. With appropriate parameters, the estimation performances of the proposed algorithm and that in [10] are close to the numerical solution. In addition, when the number of receiving antennas is small, the approximate solution is closer to the numerical solution, but the estimation error increases as the number of antennas increases.

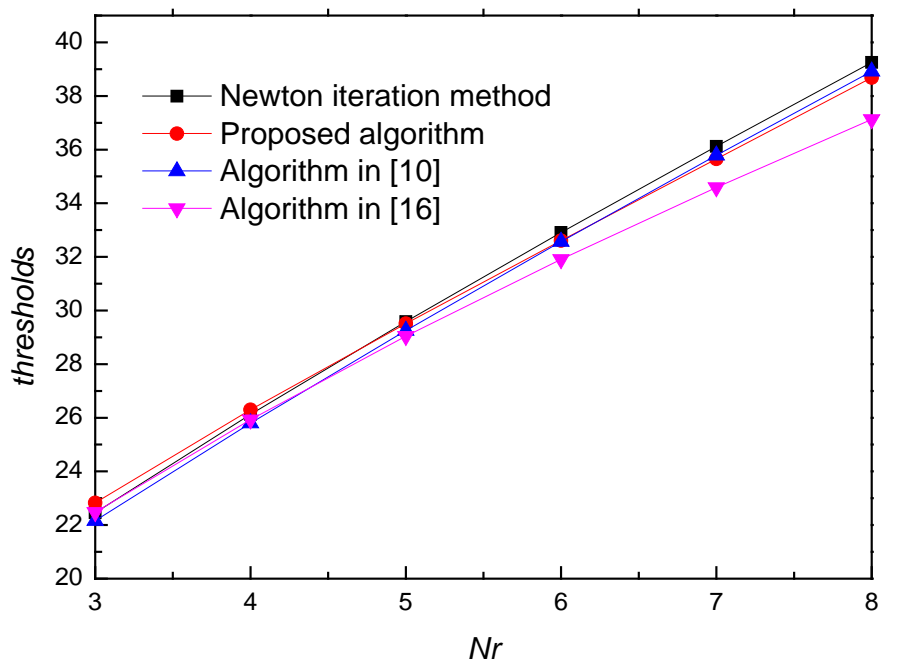

Fig. 7. Thresholds versus the number of receiving antennas for a MIMO-OFDM SFBC recognition system at the false alarm probability of $0.1 \%$.

\section{Conclusion}

In this study, an approximate analytical algorithm for the inverse of the power of the incomplete Gamma function based on EVT was investigated, which has certain theoretical and practical values. First, the power of the incomplete Gamma function was associated with the maximum limit distribution of independent and identically distributed random quantities following Gamma distributions. Then, based on EVT, the Gumbel function was used to approximate the power of the incomplete Gamma function, and the normalized coefficient of the Weibull distribution function was employed to replace the normalized coefficient of the random variable following a Gamma distribution. Finally, the approximate closed form solution was obtained. The simulation results show that when the parameters $q, \alpha$ and $n$ are appropriate, the proposed algorithm has better accuracy than those of the existing algorithms. This study also investigated the applications of the proposed algorithm in calculating the thresholds of STBC and SFBC code recognition in MIMO-OFDM. 


\section{References}

[1] Proakis, JG, "Digital Communications," Fundamentals of Codes, Graphs, and Iterative Decoding, Springer, Boston, MA, pp. 1-12, 2002. Article(CrossRef Link)

[2] Song, G., Li, Y., “Asymptotic throughput analysis for channel-aware scheduling,” IEEE Trans. Commun, vol.54, no.10, pp. 1827-1834, 2006. Article(CrossRef Link)

[3] Eldemerdash, Y.A., Dobre, O.A., Liao, B.J., "Blind identification of SM and Alamouti STBC-OFDM signals,” IEEE Trans. Wireless Commun, vol. 14, no. 2, pp. 972-982, 2015. Article(CrossRef Link)

[4] Marey, M., Dobre, O.A., "Automatic identification of space-frequency block coding for OFDM systems," IEEE Trans. Wireless Commun, vol. 16, no. 1, pp. 117-128, 2017. Article(CrossRef Link)

[5] Marey, M., Dobre, O.A., Liao, B., "Classification of STBC systems over frequency-selective channels,” IEEE Trans. Veh. Technol, vol. 64, no. 5, pp. 2159-2164, 2015. Article(CrossRef Link)

[6] Eldemerdash, Y.A., Dobre, O.A., Oner, M., "Signal identification for multiple-antenna wireless systems: Achievements and challenges,” IEEE Commun. Surv. Tutor, vol. 18, no. 3, pp. 1524-1551, 2016. Article(CrossRef Link)

[7] Karami E, Dobre O, "Identification of SM-OFDM and AL-OFDM signals based on their second-order cyclostationarity," Vehicular Technology IEEE Transactions, vol. 64, no. 3, pp. 942953, 2015. Article(CrossRef Link)

[8] DiDonato, A.R., Morris Jr, A.H., "Computation of the incomplete Gamma function ratios and their inverse,” ACM Trans. Math. Softw, vol. 12, no. 4, pp. 377-393, 1986. Article(CrossRef Link)

[9] Gil, A., Segura, J., Temme, N.M., "Efficient and accurate algorithms for the computation and inversion of the incomplete Gamma function ratios," SIAM J. Sci. Comput, vol. 34, no. 6, pp. A2965-A2981, 2012. Article(CrossRef Link)

[10] Urkowitz, H., "Hansen's method applied to the inversion of the incomplete Gamma function, with applications,” IEEE Trans. Aero. Elec. Sys, vol. AES-21, no. 5, pp. 728-731, 1985. Article(CrossRef Link)

[11] Dohler, M., Arndt, M., "Inverse incomplete Gamma function and its application,” Electron. Lett, vol. 42, no. 1, pp. 35-36, 2006. Article(CrossRef Link)

[12] De Haan, L., Ferreira, A.F, Extreme Value Theory, New York, USA: Springer, 2006.

[13] BeirlantJ, Segers J, De Waal D, Statistics of Extremes: Theory and Applications, Wiley, 2005.

[14] Gasull, A., Lopez-Salcedo, J.A., Utzet, F, "Maxima of Gamma random variables and other Weibull-like distributions and the Lambert W function,” Test: An Official Journal of the Spanish Society of Statistics and Operations Research, vol. 24, no. 4, pp. 714-733, 2015.

Article(CrossRef Link)

[15] Kalyani S., K.R.M., "The Asymptotic Distribution of Maxima of Independent and Identically Distributed Sums of Correlated or Non-Identical Gamma Random Variables and its Applications," IEEE Trans. Commun. vol. 60, no. 9, pp. 2747-2758, 2012. Article(CrossRef Link)

[16] Li, Y.; Hu, G.B., Hu, X.L., Wu, S.S., "Approximate analytic aproximation for inverse incomplete Gamma function in STBC recognition,” J. Yangzhou Uni. (Nat. Sci. Ed.), vol. 23, no. 2, pp. 36-41, 2020. Article(CrossRef Link)

[17] Gao, M., Li, Y., Dobre, O.A., Al-Dhahir, N, "Blind identification of SFBC-OFDM signals based on the central limit theorem," IEEE Trans. Wireless Commun, vol. 18, no. 7, pp. 3500-3514, 2019. Article(CrossRef Link) 


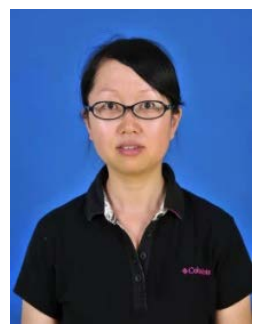

Shanshan Wu received her B.S. and M.S. degrees in electronic and information engineering from Nanjing University of Science and Technology, China, in 2004 and 2008, respectively. Currently, she is an associate professor in the School of Electronic Information Engineering, Nanjing Vocational College of Information Technology, mainly engaged in research on wireless network, cognitive radio systems, and multiple-input multiple-output antenna systems.

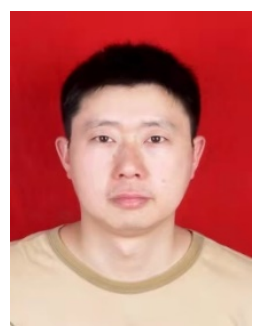

Guobing Hu received the M.S. and Ph.D. degrees in electronic and information engineering from Nanjing University of Aeronautics and Astronautics, China, in 2006 and 2011, respectively. Currently, he is a professor in the Department of Electronics and Information Engineering, Jinling Institute of Technology, and is a visiting scholar at Southeast University. His main research interests are radar signal processing and digital signal processing, with a particular focus on non-cooperative communications, cognitive radio systems, and multiple-input multiple-output antenna systems.

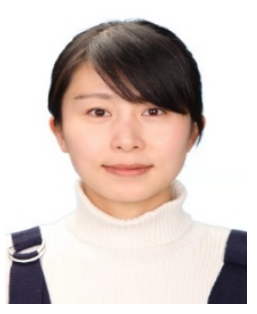

Li Yang received M. E. from Yangzhou University, Yangzhou, China, in 2008. Now she is a lab technician at Department of Electronic Information Engineering in Jinling Institute of Technology. Her research interests in radar signal processing.

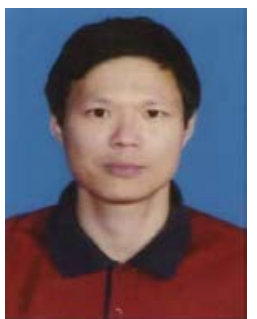

Bin Gu received his B.S., M.E., and Ph.D. degrees in electromagnetic and microwave technology from Xidian University, China, in 1989, electronic and communication engineering from Southeast University, China, in 2007, and information and communication engineering from Southeast University, China, in 2018, respectively. Currently, he is in the IoT School and the Ministerial Key Laboratory of Advanced Control for Light Industry Processes, Jiangnan University, as a researcher and also with Nanjing Vocational College of Information Technology as a professor, mainly engaged in research on wireless network and digital signal processing. 